\title{
MITIGATING FREE RIDING IN PEER-TO-PEER NETWORKS: GAME THEORY APPROACH
}

\author{
M. O. Yahaya*

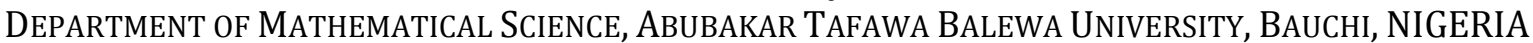 \\ Email address: mdonimisi@atbu.edu.ng
}

\begin{abstract}
The performance of peer-to-peer systems is based on the quality and quantity of resource contributions from participating peers. In most systems, users are assumed to be cooperative, but in reality, sharing in peer-to-peer systems is faced with the problem of free riding. In this paper, we model the interactions between peers as a modified gift giving game and proposed an utility exchange incentive mechanism to inhibit free riding. This technique allows peers to either upload or download resources based on their best strategy and interest. Through extensive simulations, we show that this mechanism can increase fairness and encourage resource contribution by peers to the network. This will ensure a resourceful and stable peer-to-peer systems.
\end{abstract}

Key Words: free rider, utility, peer- to-peer, game, client-server

\section{INTRODUCTION}

The unprecedented success of earlier Peer-to-Peer (P2P) applications such as KaZZaa, Gnutella, Napster has led to dramatic evolution of P2P system. Peer-toPeer (P2P) systems have been adopted as a viable alternative to client-server networks. This is due to the inadequacy of client-server architecture to cope with ever increasing demand for expansion and scalability. Servers in client-server systems sometimes constitute a bottleneck to expansion and attack on the server may results into a single point of failure. P2P system is a distributed system that eliminates partially or completely the need for a central server. A P2P system is described as a system that relies on computing power and bandwidth of nodes at the ends of a connection rather than concentrating on low number of servers within the network [1]. In [2], the authors defined P2P systems as any network that exhibits the following characteristics: distributed control, self-organized and symmetric communication. There are many types of P2P systems, mostly used for large scale content distribution, file sharing, platform sharing, communication, distributed computation and collaboration. Peers in P2P networks are autonomous - the desire to do anything without external influence. This autonomy guarantees peers independent activities; this may include voluntary sharing, free will entry and exit from the network, change of identity, honesty or dishonest dispositions to others and carry out trustworthy or untrustworthy transactions. Furthermore, peers in P2P networks have equal role. A server this time might become a client after a while. Despite these good features, P2P networks is faced with challenges of selfishness. Since contribution of resources is voluntary, some user may choose not to contribute to others if there is no incentive or enforcement, this phenomena is refers to as free riding [3]. There are several measurement studies that confirm the existence of free riders in P2P networks. In [4], the authors reported that approximately $80 \%$ of peers are free riding in a trace analysis carried out in edonkey. The results of the measurements study reported in [5] showed that 25\% of the peers in the network provide about $99 \%$ of the resources in Gnutella. Furthermore, the authors in [6, 7], found the existence of free riding in Gnutella 0.6.

Recently there are many studies exploring game theory to analyze interactions in order to inhibit free riding in P2P networks. Game theory offers a rich framework for the analysis of interactions in a strategic environment like P2P system. We found that in real P2P networks, the game model is more of one person game, in which the outcome of the game is significantly determined by the peer acting as a server as at the time of the game. In this paper, we model the interaction between peers as a modified gift giving game [8], where both interacting peers make decisions based on their strategy. This decision is 
made one at a time between the two peers with complete information.

In this paper, we propose and evaluate a simple game model, where every peer is expected to exchange its payoff with another peer it is interacting with for every upload or download in the system. This is based on the general idea that peers will not want to lose much of their utilities. A requesting peer is credited with the negative value of the current utility of the providing peer. This mechanism relies on a coordinating peer, called super peer to compute the utility exchange. This mechanism can easily be implemented in BitTorrent tracker and KaZaA overlay nodes.

The remaining part of this paper is organized as follows: Section II discusses the related work. In section III, we present the theory of gift giving game. Section IV outlined our design consideration and assumptions as well as term and definitions in this work. In section $\mathrm{V}$, we present the proposed game mechanism and analyze the operation principles of the proposed technique. In section VI, we describe the possible implementations of the model. In section VII, we explain in detail the performance evaluation, simulation set up and discusses the results of our experiment while section VIII concludes the paper.

\section{RELATED WORK}

Modeling interactions between peers in a P2P system has been examined in $[9,10,11]$. Game theory [12] has been used in the literature in an attempt to represent a peer with rational and diverse interest. Each peer in the network can be viewed as a rational game player with his own strategies to maximize his utility of the system. In strategic form of the game, the dominant strategy is not willing to share.

In [9], the authors modeled the interaction of peers in P2P network as a game theoretic model. They identified three forms of equilibrium and concluded that though social optimality of public goods cannot be reached if external incentives are not provided to the peers, some sharers will be inherently generous to provide the contents needed to maintain the networks. Hence, file sharing networks could reach equilibrium in the presence of free riding, but the degree of free riding that can be tolerated is not ascertained.

In [11], a model of incentive with strategy and Nash equilibrium in game theory is proposed. The strategy of a peer is its level of contribution and its utility is payoff which is expected benefit from other peers. The authors proposed service differentials based incentive scheme where a peer provides service to a peer based on his contribution to improve the performance of the system. They show that two equilibria exist in this form of game. Similarly, in [10], the utility function is made up of some variables such as download amount, network variety, disk space used, bandwidth, altruism and financial transfer. With the assumption that every peer is economically rational, they weigh the financial benefit and the cost of sharing. However, there is need for a fair exchange mechanism to be agreed upon by the two peers in interaction in order that the transaction ends successfully. The authors in [13] model the interactions between peers as a two player game. They presents the repeated form of the game and classified nodes as Enthusiastic, Selfish and Rational. They proposed a graded punishment for peers based on their strategy.

In [14], the researchers presented a game model of interaction between peer in a P2P systems. They study the pure strategy and mixed strategy equilibria of the game. The authors assumed all peers in the network are selfish. The contribution reputation of a peer is directly proportional to what a peer can download in a given time period. They studied the reputation changes of each peers as time progresses. The authors claimed that this inhibit free riding as free riders has to upload to be able to download equal measure. The drawbacks of this work are: the use of binary value $(0=$ no contribution in time $t, 1=$ contribution); this does not cater for the size of the resources download. Furthermore, how the service information between peers are managed is not considered.

In [15], the authors used game theory to model interaction between any two peers in an unstructured P2P system. They identified three types of peers as altruist, in-between (altruist and free riders) and free riders. They studied the strategies between two interacting altruists, two interacting free rider and between a free rider and an altruist. A modified game model that uses reputation of resource contributions of a peer is used to design what they termed "coopetion framework" to enhance cooperation among peers. The researchers used fairness index to evaluate the performance of their mechanism. Though, this work is an improvement over [14] which uses binary value for reputation mea-sure per unit time, but there are shortcomings. These includes: the ratio used to differentiate service do not discriminate effectively between peers as the sizes of files increases. Moreover, the supply of reputation information by the 
requesting peer to the providing peer is prone to manipulation. The mechanism does not provide a window for free riders to recover once their reputation goes beyond a certain predefined threshold. Other related works worth mentioning are those attempts in literature to model resource sharing in P2P networks with ideas from Evolutionary Game Theory.

In [16], the authors modeled the resource sharing in P2P with Evolutionary game theory with the claim that Nash equilibrium is hard to compute and does not capture the highly dynamic nature of P2P networks and the researchers in [17] proposed a model _le caching in P2P networks using micro-macro system dynamics and Evolutionary game theory. They assumed that every node is aware of the behaviour of all other nodes in the system. In [18], the author proposed that one can use Evolutionary game theory to measure P2P system robustness. However, the requirement that players pass on their strategies to their off-springs as time goes on does not correctly capture peers characteristics. Since, in P2P systems every peer is an autonomous entity on its own. The assumption that every player has the knowledge of the whole system is not realistic. The possibility of one strategy displacing other strategy with time no matter how high the payoff is not possible. Furthermore, at micro level interaction between peers, the strategy set is not so dynamic. Moreover, the use of Evolutionary game theory considers the dynamics of all the nodes in the network at time. This is not suitable to model peers interaction which always reduced to a repeated form of two players game.

\section{GIFT-GIVING GAME}

In gift giving game model [8], players are randomly selected and match to play a game in a large population $n$. Each players is assumed to have information of the past behaviour of his opponent. In [19], the author provided this assumption that this information is distributed by what he termed as 'information system' The game assumptions are as follows: (1) There are two types of agent named young and old (2) Each agent lives for two periods (3)There are equal number of each agent type alive in every time period.

Table 1: Payoff matrix for gift giving game

\begin{tabular}{|l|l|l|}
\hline & Young/Give & Young/Keep \\
\hline Old & $(\alpha, 1)$ & $(0,0)$ \\
\hline
\end{tabular}

The game is described as follows: The objective of every player is to maximize his payoff in both time period. In the first time period the player is referred to as young and in the second period it is referred to as old. Each young player is matched with an old player to play a game. This young player must choose between a gift of 0 or 1 . The old player has no decision to make. But if the young player select gift of value 0 , both player receive a payoff of 0 ; if he chooses a gift of value 1 , he gets a payoff of -1 and the old player receive a payoff $\alpha>1$. The lifetime utility is the sum of utilities over the two periods of life. The payoff matrix of this game is shown in Table 1

\section{ASSUMPTIONS}

In this section, we listed all the assumptions we made in the design of our system

(i) All peers in the networks can serve as either a resource provider (target peer) or the resource requester (source peer) except the super peers. The functions of the super peer is to manage the update and information exchanges between ordinary peers.

(ii) All the ordinary peers in the networks have equal upload and download capacity.

(iii) To make the problem tractable, the size of all resources in the networks are equal and fixed.

(iv) Each peer in the network has a resource to provide and may also request for resources from others, and they all have equal number of resources.

(v) A particular resource can be owned by many peers, so that a requesting peer can select from the candidate providers based on the peers utility objectives.

\subsection{Definition and terms}

- Strategy: set of best choices in the game. That is a layout plan of actions for the entire game. The term strategy and action are used interchangeably.

Utility: This is the net gain, that is, the difference between incentives and cost incurred in providing service. It is also referred to as payoff in this paper.

- Requesting peer: This refers to the peer acting as a client at that time. It may also be refer to as source peer or requester in this work.

Providing peer: This refers to the peer acting as a server at that time. It is sometimes called target peer or provider in this work. 


\section{THE PROPOSED P2P SHARING GAME MODEL}

\subsection{Model descriptions}

Consider a file sharing P2P networks in which two peers $p 1$ and $p 2$ are to share a file. Every user is rational and is aware of his preference and consequences of their actions. In a game, nodes request service for themselves, and decide whether to serve others or not.

The players in this game are classified into three namely: Free riders - These are set of players that adopt selfish strategy with probability of sharing equals zero. They never provide resources to any peer throughout their stay in the network. In-between peers are the class of players that have approximately $50 \%-50 \%$ chance of sharing or not sharing depending on the provider and the scenario. Altruist are set of players that share all the time, such that, the probability of sharing equal 1 . Hence, the game model consist of:

- $\quad$ Set of players $=\{$ Freeriders, In-between, Altruist $\}$

- $\quad$ Set of Actions $=\{$ Share, Not share $\}$

- $\quad$ Payoff for each player $=U$

Let's consider a file of the same size or file chunk of same size. We consider the payoff as dimensionless functions depending on size of file or chunk, download priority, download speed and download options. The cost function can be a function of bandwidth utilized and disk space used in providing the resource. In reality, it is difficult to compute the values of this function since some are subjective.

Let $C U t$ be the current utility derived from the system by peer $i$ at time $t$. If peer $i$ receives a resource from peer $j$, It cost the receiving peer $i$ an equivalent of the current utility of the providing peer $j$. In that case, the payoff is $(U j)$. Conversely, peer $\mathrm{j}$ gains an equivalent of the current utility of peer $i$. That is the payoff is $(+U i)$. At the end of every time period, the utility $(U)$ of every peer is updated according to the rule above by the super peer as shown in equation 1 .

$C U t=C U t 1+U$

In (1), CUt is the cumulative utility at time $t$ and $U$ is the current utility (payoff). We assume that peers only give when the benefit - cost $>0$. Let $U i$ be the utility derived by peer $i$, that is net gain which is the difference of benefit and the cost. Let $f 1(:)$ denotes the function of benefit derived by peer $i$ from an object of size S. Let $g 1(:)$ be the function of cost for uploading an object of Size i. Hence, $U=f 1(:) \quad g 1(:)$. The utility (payoff) matrix of the game is shown in Table 2. This is a modified form of a gift giving model in [20]. We incorporate free riding mitigation capability into the game using the utility exchange mechanism described earlier. We divided the game-based mechanism into two stages.

Table 2: Payoff matrix for the players

Share

Provider Utility $(U p)$, Requester Utility $(U r)$

Not Share 0,0

Table 3: Sample list returned to requester

\begin{tabular}{|l|l|l|}
\hline ResourceID & OwnersID & $\begin{array}{l}\text { Current } \\
\operatorname{Utility}(U t)\end{array}$ \\
\hline & & \\
\hline
\end{tabular}

\subsection{Stage 1: Resource providers' selection procedure}

When a peer Pi needs a resource from the network, the source peer (requesting peer) send a request message for a particular resource to the super peers it is connected to in the network. The super peer returns the list of all the peers that has the requested resource to the requester and their utility as shown in Table 3 . This list contains the resourceID, the ownersID and their utilities. The requesting peer has to take the best decision in selecting the provider of its desired resource. The requesting peer scans through the list of all owners of that resource starting from the provider with the lowest utility to the provider with the highest utility. This procedure is to minimize the level of reduction to the requesters' utility. Since, the rationality assumption in game theory is that every peer will want to maximize its utility from the system. Except for Altruist, who do not care sometimes about the gain from the system as far as the cost of providing is minimal. In case, the requesting peer decides not to choose from the list of providers, the peer abandons this game and makes another request. The game continues until the peer downloads the desired resource or there are no providers willing to give or it leaves the networks.

\subsection{Stage 2: Providers decision procedure}

The providing peer once selected by the requesting peer takes it turn in the game to make a move. It is provided with the current utility of the requesting peer. The peer decides its best strategy to serve or not based on the expected gain which is proportional to the current utility of the requester. The exchange of resources is completed once the provider agrees to serve. Then, the super peer updates their record and the game continues. 


\subsection{Free riders mitigation mechanism analysis}

In this subsection, we analyze the operations of this mechanism. In every trans-action, the game is a two player game, that is, a two peer game between the providing peer and requesting peer. The possible interactions between two peer types based on the mechanism are shown in Table 4. Each peer has different utility based on their characteristics. For example, the payoff of an Altruist is always high. If a free riding peer wants a resource from an Altruist, it suffers a negative payoff because, it costs the requester, an equivalence of the total payoffs of the providing peer. The requesting peer will prefer to choose a peer with lower payoff so as to incur lesser cost. With this idea, the advantages of this mechanism are as follows: (1) Altruist in the system will not be overwhelmed by free riders. This is due to fact that free riders search from low to high since requesting from altruist costs free riders more. Hence, fairness index is close to unity (1). (2) The mechanism gives chance to willing In-between peers to increase their utility by providing more to others. The notation in Table 4 are as follows:

- $\quad=$ peers with current negative utility

$+=$ peers with current positive utility

$\mathrm{F}=$ Free rider

$\mathrm{I}=$ In-between

$\mathrm{A}=$ Altruist

or example, if I = (In-between) is the provider and A $=$ (Altruist) is the requester, then the pair $(+,+)$ means that both requester and provider have positive current payoffs and the probability of resource exchange is high, because the provider gains in this transaction.

\section{MODEL IMPLEMENTATION}

In the following subsections, we describe in details the possible implementations of this model.

\subsection{System architecture}

The concept of peer-to-peer networks is same, but there are different implementations. They can be classified based on different criteria such as structure, degree of centralization, type of provided services, and access level by participants. The design of any P2P networks can be based on any of these classifications. In this model, we consider a hybrid unstructured P2P networks where there exists super nodes that coordinates the activities of ordinary nodes connected to it. In this game, the super nodes handle the management and exchange of utilities information of every peer connected to that super node. For instance, the overlay node in KaZaA that manages the contribution level of every peer connected to it. Similarly, the tracker in BitTorrent, in addition to its functionality of providing the peers with the requested resources can also serve as the manager of the share history and utility of every peers connected to it.

In this model, only one request will be processed by the tracker in a single time period. The tracker has a central table of requesters and candidate providers in the system as shown in Table 5. It chooses one request at a time on First Come First Serve basis to be processed. For each resource request, a list of candidate providers as depicted in Table 6 is processed for each request by the tracker.

Table 4: Mechanism Operations Analysis

\begin{tabular}{|c|c|c|}
\hline Provider & Requester & $\begin{array}{l}\text { Service Probability and } \\
\text { Comment (Reason) }\end{array}$ \\
\hline A & A & $\begin{array}{l}(+,+), \text { satisfied, both are } \\
\text { altruists. Current utilities are } \\
\text { positive }\end{array}$ \\
\hline A & I & $\begin{array}{l}\text { Case 1: }(+,+) \text {, probability of } \\
\text { serving is high, because } \\
\text { provider gains }\end{array}$ \\
\hline A & I & $\begin{array}{l}\text { Case 2: }(+,-) \text {, probability of } \\
\text { serving is low, provider looses }\end{array}$ \\
\hline A & $\mathrm{F}$ & $\begin{array}{l}(+\quad-) \text { No service will be } \\
\text { provided, provider looses }\end{array}$ \\
\hline I & A & $\begin{array}{l}\text { Case 1: }(+,+) \text {, probability of } \\
\text { serving is high, because } \\
\text { provider gains }\end{array}$ \\
\hline I & A & $\begin{array}{l}\text { Case 2: }(+,-) \text {, probability of } \\
\text { serving is low, provider looses }\end{array}$ \\
\hline I & I & $\begin{array}{l}\text { Case } 1:(+,+) \text {, probability of } \\
\text { serving is high, because } \\
\text { provider gains }\end{array}$ \\
\hline I & I & $\begin{array}{l}\text { Case 2: }(+,-) \text {, probability of } \\
\text { serving is low, provider looses }\end{array}$ \\
\hline I & I & $\begin{array}{l}\text { Case 3: }(-,+) \text {, probability of } \\
\text { serving is low, provider looses }\end{array}$ \\
\hline I & I & $\begin{array}{l}\text { Case 4: }(-,-) \text {, probability of } \\
\text { serving is low, provider looses }\end{array}$ \\
\hline I & $\mathrm{F}$ & $\begin{array}{l}\text { Case 1: }(+,-) \text {, probability of } \\
\text { serving is low, provider looses }\end{array}$ \\
\hline I & $\mathrm{F}$ & $\begin{array}{l}\text { Case 2: }(-,-) \text {, no service will be } \\
\text { provided, provider looses }\end{array}$ \\
\hline $\mathrm{F}$ & A & $\begin{array}{l}(-,++), \text { no service will be } \\
\text { provided, free riders never } \\
\text { gives }\end{array}$ \\
\hline $\mathrm{F}$ & I & $\begin{array}{l}\text { Case } 1 \text { and case } 2:(-,-) \text { and }(+,- \\
\text { ) no service will be provided, } \\
\text { free riders never gives }\end{array}$ \\
\hline $\mathrm{F}$ & $\mathrm{F}$ & $\begin{array}{l}(-,-), \text { no service will be } \\
\text { provided, free riders never } \\
\text { gives }\end{array}$ \\
\hline
\end{tabular}




\subsubsection{Two-peer Interactions Sequence}

Consider a file sharing interaction between two peers $P x$ and $P y$. The interaction steps are as follows:

Step 1: $P_{X}$ sends a download request to the server or super peer for a specific file.

Step 2: The super peer selects candidates' peers with the requested file. The selection is carried out by the server or super peer based on the utility of $P_{X}$. That is based on its type; be it Free rider, In-between or Altruists.

Step 3: Assume Py is among the list provided by the super peer to $P_{X}$ with their respective utilities as in Table 6. The selection from the list by $P_{X}$ could be random or sequential depending on $P X^{\prime}$ s objective.

Step 4: $P y$ agree to serve $P_{X}$ or not based on $P y$ 's strategy. If $P y$ agree, the file is transferred to $P X$.

Step 5: Update of both peers utility is carried out by the server or the super peer.

Note that we are assuming that both peers are provided with information, honest and trustworthy.

Table 5: Centralized game model

\begin{tabular}{|c|c|c|c|c|c|}
\hline & $S 1$ & $S 2$ & 53 & $\ldots$ & $S n$ \\
\hline$T 1$ & $(+U s 1, U T 1)$ & $(+U s 2, U T 1)$ & (+Us3;UT1) & $\ldots$ & (+Usn;UT1) \\
\hline$T 2$ & $(+U s 1, U T 2)$ & $(+U s 2, U T 2)$ & (+Us3;UT2) & $\ldots$ & (+Usn;UT2) \\
\hline$\dot{T_{n}}$ & $(+U s 1, U T m)$ & $(+U s 2, U T m)$ & $(+U s 3 ; U T m)$ & ... & $(+U s n ; U T m)$ \\
\hline
\end{tabular}

\section{Performance Evaluation}

In the next two subsections, we describe the performance metrics used to evaluate the proposed model and detailed explanation of simulation steps and parameters.

Table 6: A file request game model

\begin{tabular}{cc}
\hline & S1 \\
\hline$T 1$ & $(+$ Us $1 ;$ UT1 $)$ \\
$T 3$ & $(+$ Us 1 UT1) \\
$\cdot$ & \\
$\cdot$ & \\
Tm & $($ Us $1 ;$ UTm $)$ \\
\hline
\end{tabular}

\subsection{Performance metrics}

The objectives of any free riding mitigation mechanism are:

(1) To ensure cooperation among participating peers

(2) To ensure fairness amongst all peers in the system and

(3) To alleviate the impact of unauthentic and malicious resources in the system. In this paper, we defined free riding as those peers that do not contribute resources to other peers in the system. Hence, we evaluate the performance of our mechanism with the performance evaluation criteria proposed in [15]. The criteria are as follows:

(1) the number of requests fulfilled by free riders: this measure the number of successful requests peers get from other peers in the network considering the behaviour of that peer.

(2) the ratio of upload to download of each peer in the system (fairness index): This measures the fairness of the whole system to the peers in terms of resource provision characteristics. For instance, we do not want few Altruists in the system to be overwhelmed by the requests of many free riders in the system. We also prefer each peer get resources proportional to its contributions to others in the system. The fairness index formula is given as

$$
\text { Fairness Index }=\frac{\left(\sum_{i=1}^{n} x_{i}\right)^{2}}{n \sum_{i=1}^{n} x_{i}^{2}}
$$

In (2), $x_{i}$ is defined as the ratio of upload to download of every peer. The fairness index is bounded by 0 (unfair) and 1 (fair). The larger the value the fairer the whole network [15]. The fairness index of 1 does not exists in real system due to availability of free riders. Assume there are $N s$ of peers in the networks, $N_{f}$ Free riders, $N i$ In-between, and $N a$ Altruists with ratios $\mathrm{P}_{\mathrm{f}} ; \mathrm{P}_{\mathrm{f}}$ and $P a$ respectively. The value $X i$ for free riders is 0 , hence, the maximum fairness index will be a constant $Q$ and it is bounded by the ratio of free riders. This can be derived as follows:

$$
\begin{aligned}
\text { Fairness Index } & =\frac{\left(P_{f} \cdot N_{s} \cdot 0+\left(1-P_{f}\right) \cdot N_{s} \cdot Q\right)^{2}}{N_{s}\left(P_{f} \cdot N_{s} \cdot 0^{2}+\left(1-P_{f}\right) \cdot N_{s} \cdot Q^{2}\right)} \\
& =1-P_{f}
\end{aligned}
$$

Table 7: Simulation and system parameters

\begin{tabular}{lll}
\hline Parameters & Description & value \\
\hline$T s$ & $\begin{array}{l}\text { The number of } \\
\text { simulation time }\end{array}$ & 100 \\
& The number of & 1000 \\
$N r$ & The number of & 40000 \\
$N c$ & The number of & 10 \\
$\Lambda$ & $\begin{array}{l}\text { Arrival rate of } \\
\text { requests }\end{array}$ & 3 \\
Peer Type I & Free rider (Never & $\mathrm{P}($ share $)=0$ \\
Peer Type II & share) & $\mathrm{P}($ share $)=0.5$ \\
Peer Type III & $\begin{array}{l}\text { In-between } \\
\text { (Sometimes share) }\end{array}$
\end{tabular}


Table 8: Simulation Combination

\begin{tabular}{|c|c|c|c|}
\hline Peer Type & \multicolumn{2}{|c|}{ Free rider In-between } & Altruists \\
\hline Ratio 1 & $33 \%$ & $33 \%$ & $33 \%$ \\
\hline Ratio 2 & $70 \%$ & $20 \%$ & $10 \%$ \\
\hline Ratio 3 & $10 \%$ & $70 \%$ & $20 \%$ \\
\hline Ratio 4 & $10 \%$ & $20 \%$ & $70 \%$ \\
\hline Ratio 5 & $0 \%$ & $30 \%$ & $70 \%$ \\
\hline Ratio 6 & $70 \%$ & $30 \%$ & $0 \%$ \\
\hline
\end{tabular}

\subsection{Simulation setup}

The goal of the simulation is to evaluate the performance of the proposed mechanisms. The simulation model is based on other P2P studies that simulate P2P file sharing networks such as in [21], [22], and [15]. We simulate our model using PeerSim [23], a P2P simulator and BitPeer [24] BiTorrent module for Peersim-to evaluate the performance of our proposed method. We utilized the tracker in the BitTorrent module to test our algorithm. The simulation parameters are as shown in Table 7.

We varied the ratio of different classes of peers in the network and test the simulation combination as shown in Table 8 . The simulation has the following phases: the initialization phase, request generation, the game and computation of fairness index. During

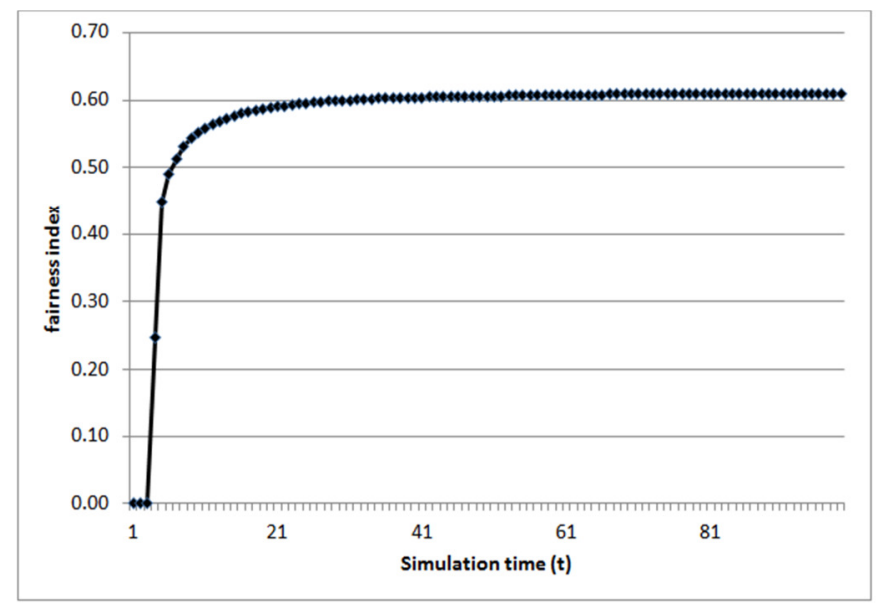

Figure 1: Fairness index Vs Simulation Time ( $F=33.3 \%$, $I=33.3 \%, A=33.3 \%$ )

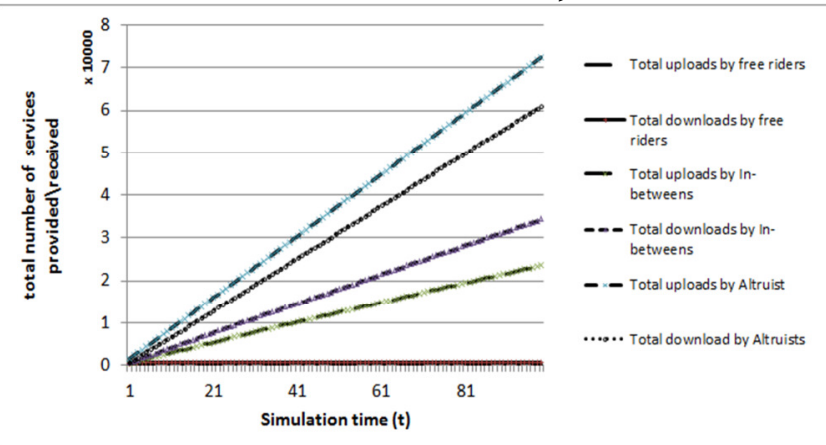

Figure 3: Total number of service provided and received per peer Vs Simulation Time $(t)(F=33.3 \%, I=33.3 \%$, $A=33.3 \%)$ initialization, resources are distributed evenly to all peer types. A copy of a resource may be with several peers. In every time slot, resources request arrives from peers using Poisson distribution with mean $\lambda$. The tracker picks the request and processes it.

We initialize the system by assigning initial payoff (utility) of 1 unit to every peer in the system to start with. As interaction among peers goes on, the payoff of each downloading or uploading peer is updated according to the model. At the end of every time slot, the following values are computed for each peer, the ratio of upload to download, the number of request(s) provided and received. The results are discussed in the next section.

\section{RESULTS AND DISCUSSIONS}

\subsection{Experiment 1}

In the first set of experiments, we test equal composition of peer type (Freerider $=33.3 \%$, Inbetween $=33.3 \%$, Altruist $=33.3 \%$ ) to investigate the effect of time on fairness of the network. We measure the fairness for simulation time for 100 and 200 time slot. Each event occurs in a time slot and the length of one time slot is $1000000 \mathrm{~ms}$.

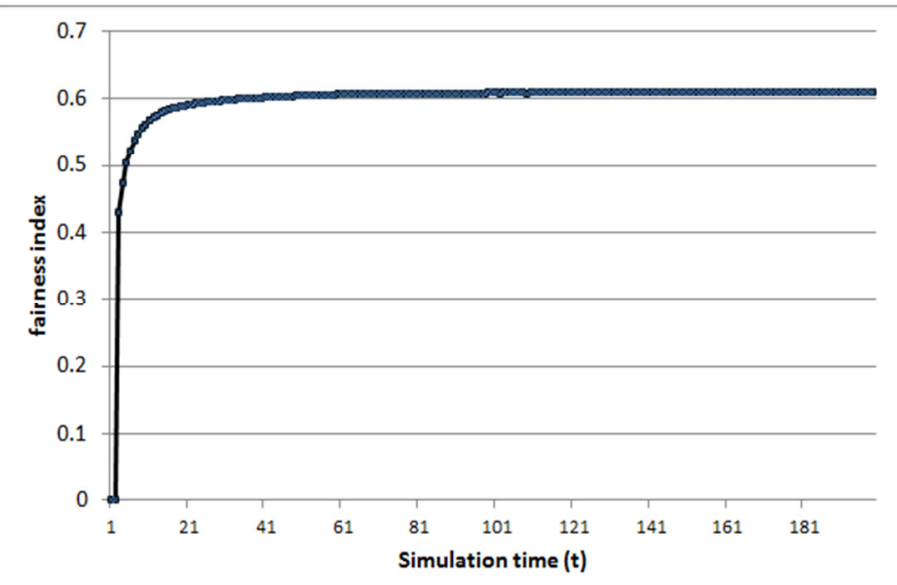

Figure 2: Fairness index Vs Simulation Time ( $F=33.3 \%$, $I=33.3 \%, A=33.3 \%$ )

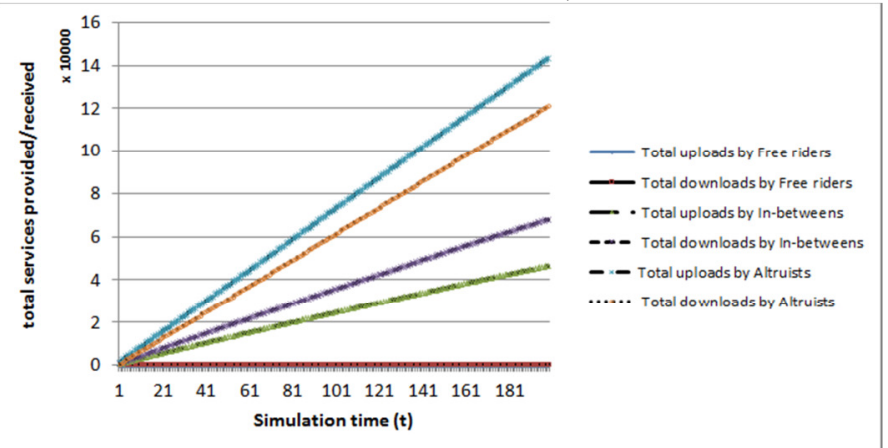

Figure 4: Total number of service provided and received per peer $V$ S Simulation Time $(t)(F=33.3 \%, I=33.3 \%$, $A=33.3 \%$ ) 


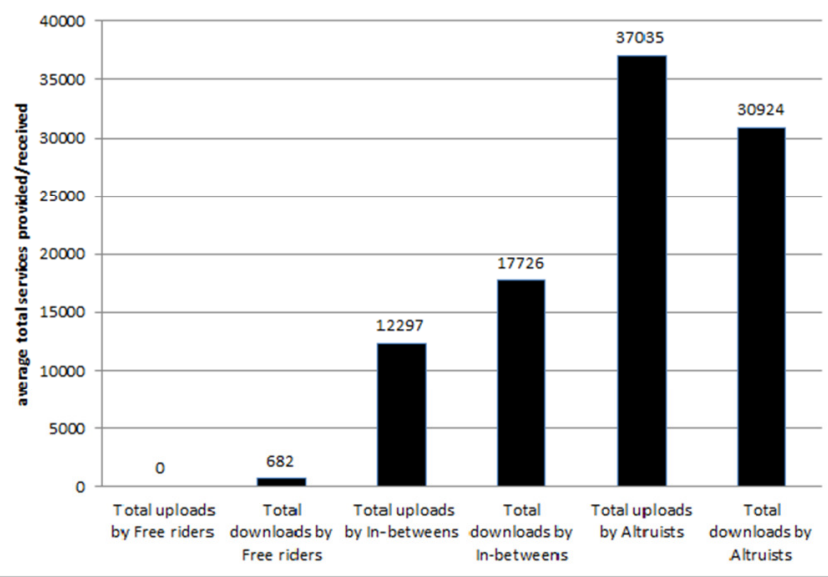

Figure 5: Average total services provided/received VS Peer type $(t=100)$

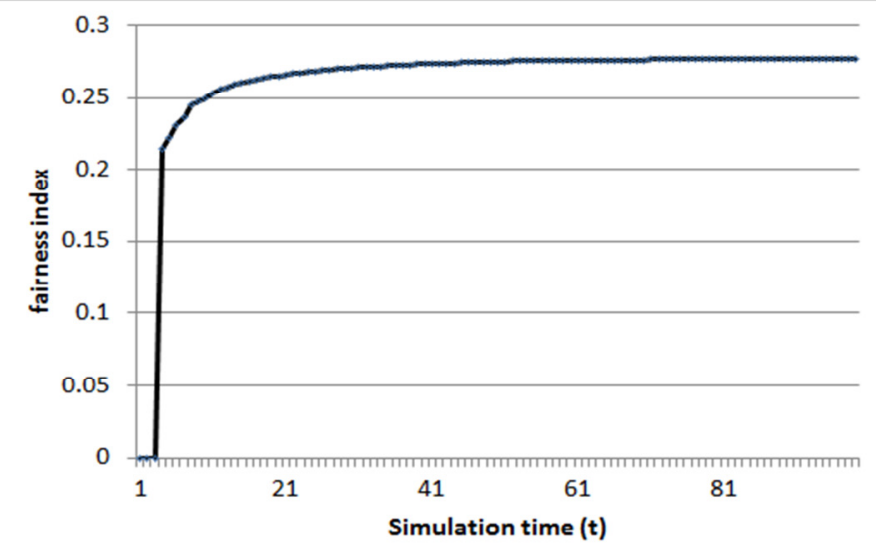

Figure 7: Fairness index Vs Simulation Time ( $F=70 \%$, $I=20 \%, A=10 \%$ )

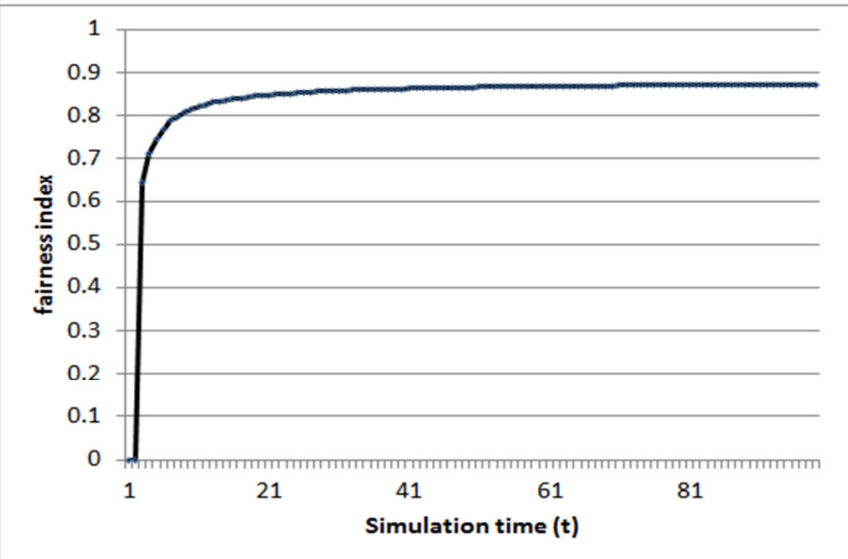

Figure 9: Fairness index Vs Simulation Time ( $F=10 \%$, $I=70 \%, A=20 \%$ )

We observe that the system is stable after a short time of around 20 unit of simulation time. The results are shown in Figures 1, 2, 3 and 4. We also notice that for the peer type Altruist and In-between, the total number of resources provided is proportional to the resource received in the networks. Though, we clearly see that the resources provided by Altruists are slightly higher than what is received, that is expected based on their characteristics. That is, $\mathrm{P}$ (sharing) $=1$. Although In-between peers get slightly higher than

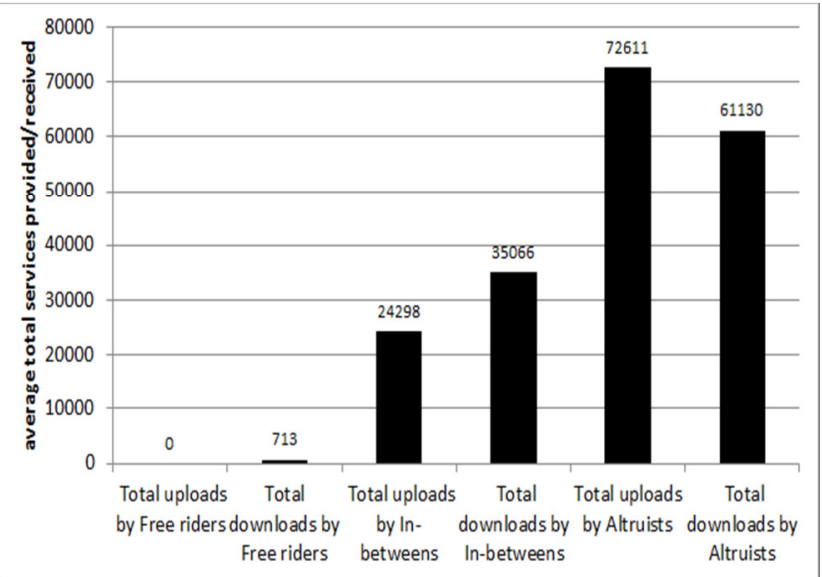

Figure 6: Average total services provided/received Vs Peer type $(t=100)$

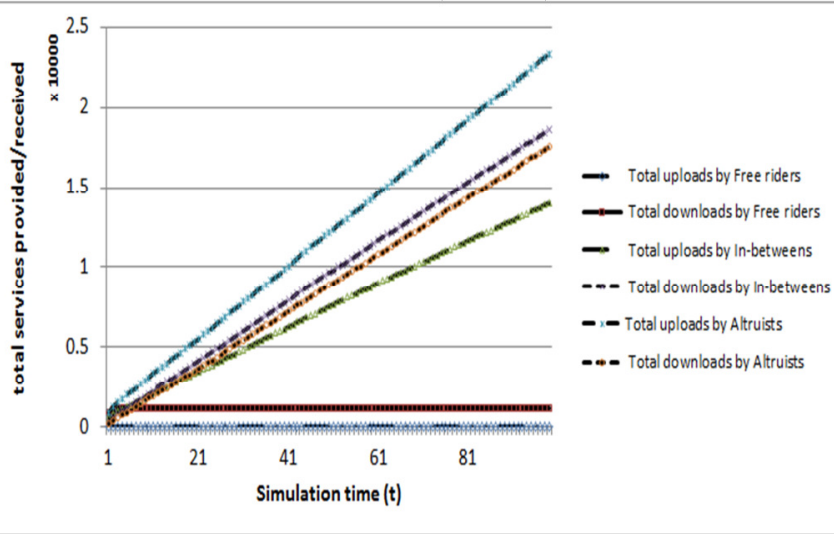

Figure 8: Total number of service provided and received per peer Vs Simulation Time $(t)(F=70 \%$, $I=20 \%, A=10 \%$ )

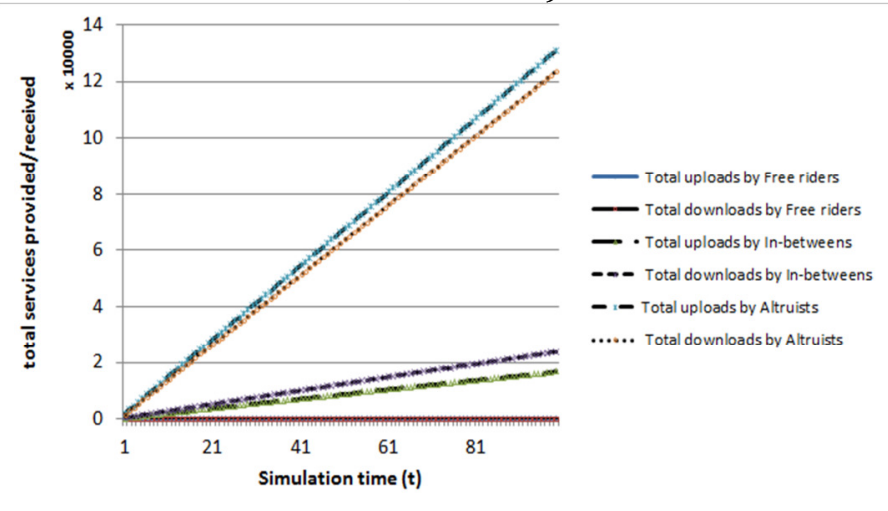

Figure 10: Total number of service provided and received per peer Vs Simulation Time $(t)(F=10 \%$, $I=70 \%, A=20 \%$ )

their contributions, this does not affect overall fairness in the long run.

For free riders that do not contribute to any peer in the system. They get on the average very low number of services and get isolated from the network. This shows the effectiveness of our proposed mechanism. The relative average service provided/ received by each peer type is shown in Figures 5 and 8 for simulation time of 100 and 200 respectively. 


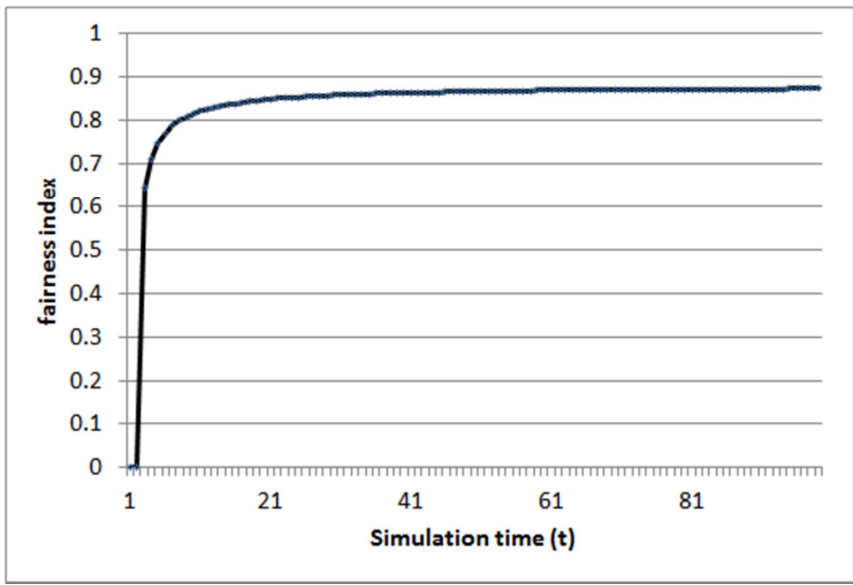

Figure 11: Fairness index Vs Simulation Time $(F=10 \%$ $I=20 \%, A=70 \%$ )

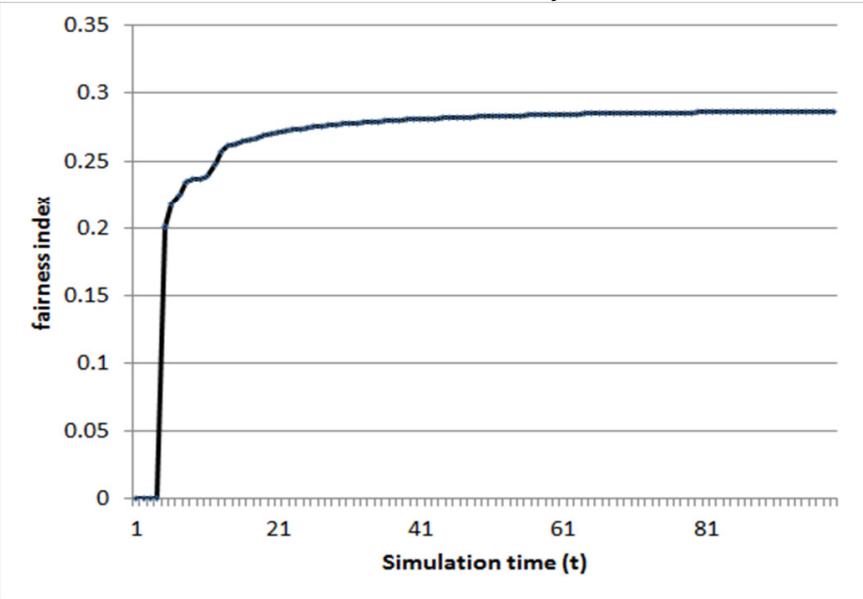

Figure 13: Fairness index Vs Simulation Time ( $F=70 \%$ $I=30 \%, A=0 \%$ )

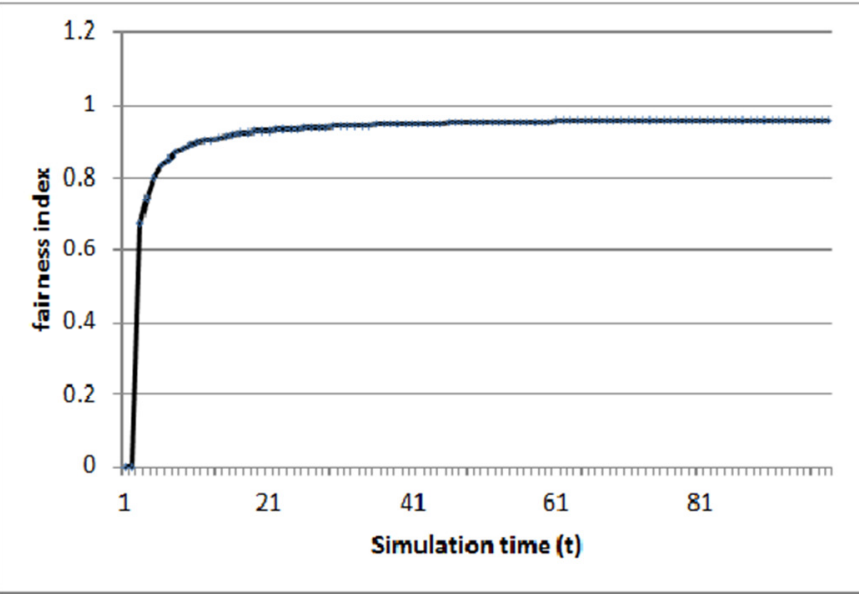

Figure 15: Fairness index Vs Simulation Time ( $F=0 \%$, $I=30 \%, A=70 \%$ )

In Figures 8 and Figure 14 show where free riders dominate the network with ratio $70 \%$ compared to other peer type between $0-30 \%$. It is interesting to note that the service received is very minimal throughout the whole network lifetime. Hence, we conclude that our mechanism is effective in mitigating the effect of free riding and by isolating them from service consumption from the network as far as they

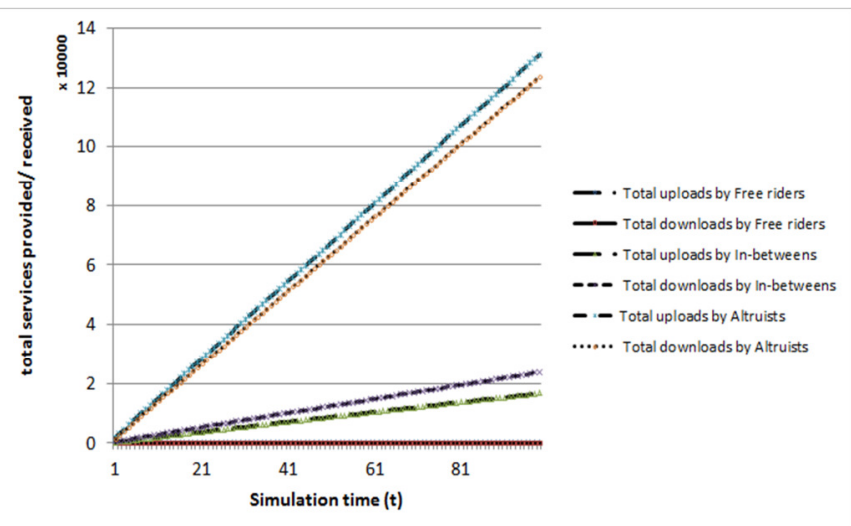

Figure 12: Total number of service provided and received per peer $V s$ Simulation Time $(t)(F=10 \%$, $I=20 \%, A=70 \%$ )

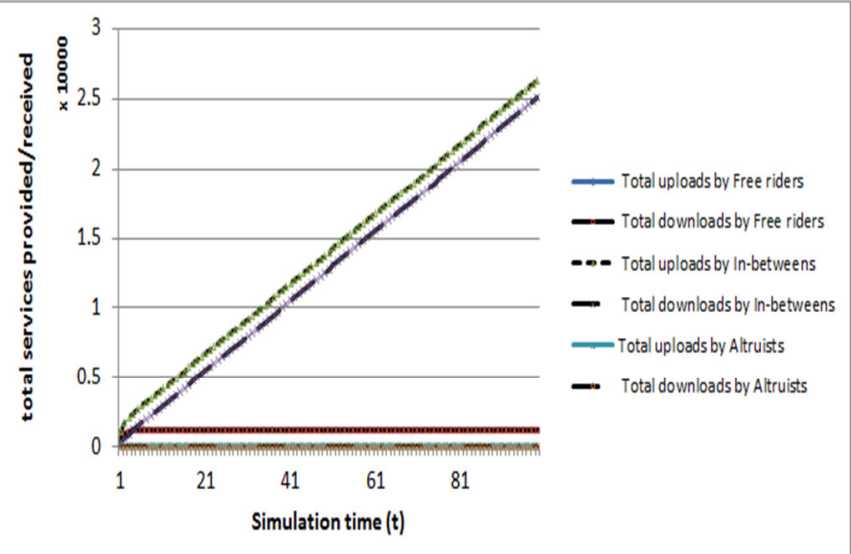

Figure 14: Total number of service provided and received per peer Vs Simulation Time $(t)$ ( $F=70 \%$, $I=30 \%, A=0 \%)$

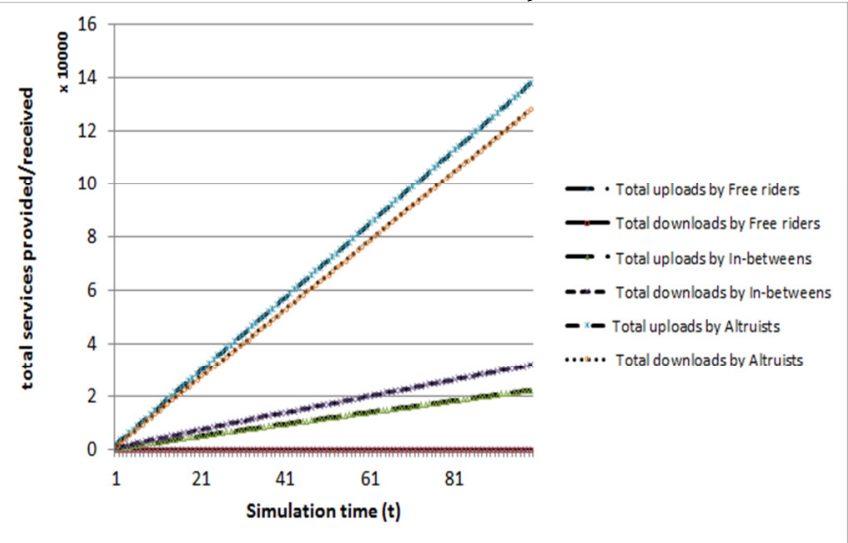

Figure 16: Total number of service provided and received per peer Vs Simulation Time $(t)(F=0 \%$, $I=30 \%, A=70 \%$ )

did not contribute resources to others.

\subsection{Experiment 2}

We repeat the experiment for a fixed simulation time, but vary the ratio of the composition of the peer type. This is to measure the impact of the number of peer type to our mechanism and the network. We observed that the fairness is stable as shown in Figures 7,9, 13, 
15 and 17.

As expected, the cumulative number of service received and provided by Altruist and In-between peers where they dominate the system is higher, as shown in Figure 12, Figure 16 and Figure 10.

\section{CONCLUSION AND FUTURE WORK}

We have proposed a theoretic game mechanism for mitigation of free riding in $\mathrm{P} 2 \mathrm{P}$ networks. We have demonstrated through extensive simulations the effectiveness of our proposed mechanism. However, this paper only considered free riding and assumed all peers are obedient and trustworthy. We did not consider cases of peers that may contribute unauthentic resources to elicit services from other and also, some peers that might share malicious content or being dishonest in their transactions. As part of our future work, we will consider incorporating mechanism to isolate peers that share unauthentic and malicious resources in the network.

\section{REFERENCES}

1. P.Pradeep, N. Kumar, R. S. Shekar, Reddy, and C. Krishna, "Preventive measures for malware in p2p networks." International Journal of Engineering Research and Applications (IJERA), vol. 2, no. 1, pp. 391-400, Feb. 2012.

2. M. Roussopoulos, M. Baker, and D. S. H. Rosenthal, "2 P2P or Not 2 P2P ?" in IPTPS'04 Proceedings of the Third internationa conference on Peer-to-Peer Systems, 2004, pp. 1-6.

3. M. Feldman and J. Chuang, "Overcoming free-riding behavior in peer-to-peer systems," ACM SIGecom Exchanges, vol. 5, no. 4 , pp. $41\{50$, Jul. 2005. [Online]. Available: http://portal.acm.org/citation.cfm?doid=1120717.11207 23

4. S. B. Handurukande, A.-M. Kermarrec, F. Le Fessant, L. Massouli_e, and S. Patarin, "Peer sharing behaviour in the edonkey network, and implications for the design of server-less file sharing systems," SIGOPS Oper. Syst. Rev., vol. 40, no. 4, pp. 359-371, Apr. 2006

5. E. Adar and B. A. Huberman, "Free Riding on Gnutella," First Monday, pp. 1-22, 2000

6. A. Asvanund, K. Karen, Clay. Ramayya, and D. S. Micheal, "An Emperical Analysis of Network externalities in Peer-toPeer Music Sharing networks," Information System Research, vol. 15, no. 2, pp. 155-174, 2004.

7. S. Saroiu and P. Gummadi, "A measurement study of peer-topeer file sharing systems," in Multimedia Computing and Networking, 2002.

8. J. Philip and D. K. Levine, "Evolution and information in a giftgiving game," Journal of Economic Theory, vol. 100, pp. 121, 2001.

9. R. Krishnan, M. Smith, and Z. Tang, "The impact of free-riding On peer-to-peer networks," in the 37th Annual Hawaii International Conference on System Sciences, 2004, pp. 1-
10.

10. P. Golle, K. Leyton-brown, I. Mironov, and M. Lillibridge, "Incentives for Sharing in Peer-to-Peer Networks," Electronic Commerce, 2001.

11. C. Buragohain and D. Agrawal, "A game theoretic framework for incentives in P2P systems," in International conference on Peer-to-Peer Computing, vol. 93106, no. 1, 2003, pp. 4556.

12. M. J. Osbourne, An Introduction to Game Theory. Oxford University press, 2003.

13. W. Li, J. Chen, and B. Zhou, "Game theory analysis for graded punishment mechanism restraining free-riding in $\mathrm{p} 2 \mathrm{p}$ networks," in Proceedings of the 2011 International Symposium on Computer Science and Society, ser. ISCCS '11. Washington, DC, USA: IEEE Computer Society, 2011, pp. 262-266.

14. R. Gupta and A. K. Somani, "Game theory as a tool to strategize as well as predict nodes behavior in peer-topeer networks," in Proceedings of the 11th International Conference on Parallel and Distributed Systems - Volume 01, ser. ICPADS '05. Washington, DC, USA: IEEE Computer Society, 2005, pp. 244-249.

15. J. S. Hua, D. C. Huang, S M Yen, and C. W. Chena, "A dynamic game theory approach to solve the free riding problem in the peer-to-peer networks," Journal of Simulation, vol. 6, pp. $43-55,2012$.

16. Q. Zhang, H.-F. Xue, and X. dong Kou, "An evolutionary game model of resources-sharing mechanism in p2p networks," in Proceedings of the Workshop on Intelligent Information Technology Application (IITA'07), Zhang Jiajie, China, 2-3 December, 2007, 2007, pp. 282-285.

17. Y. Matsuda, M. Sasabe, and T. Takine, "Evolutionary game theory-based evalu-ation of $\mathrm{p} 2 \mathrm{p}$ file-sharing systems in heterogeneous environments," Int. J. Digital Multimedia Broadcasting, 2010.

18. B. Q. Zhao, J. C. S. Lui, and D.-M. Chiu, "A mathematical framework for analyzing adaptive incentive protocols in p2p networks," IEEE/ACM Trans. Netw., vol. 20, no. 2, pp. 367-380, Apr. 2012.

19. G. Kandori, M Mailath and R. R., "Learning, mutations and Long run equilibria in games," Econometrica, vol. 61, pp. 27-56, 1993.

20. Z. Yu and n. Mihaela vander Schaa, "Peer-to-peer multimedia sharing based on social norms," SignalProcessing: Image Communication, vol. 27, pp. 383400, 2012.

21. S. Kamvar and M. Schlosser, "The eigentrust algorithm for reputation management in p2p networks," in $W W W{ }^{\prime} 03$ Proceedings of the 12th international conference on World Wide Web, 2003.

22. M. Zghaibeh, "A Lottery-based pricing scheme for peer-topeer networks," in IEEE International Conference on Telecommunication Systems, 2008, pp. 903-908

23. A. Montresor and M. Jelasity, "PeerSim: A scalable P2P simulator," in Proc. of the 9th Int. Conference on Peer-toPeer (P2P'09), 2009.

24. F. Fabrizio and M. Pedrolli, "A bittorrent module for peersim," in University of Trento, 2008. 